\title{
Priestley duality for N4-lattices
}

\author{
Ramon Jansana ${ }^{1}$ Umberto Rivieccio $^{2}$ \\ ${ }^{1}$ Department of Logic, History and Philosophy of Science, University of Barcelona, Spain \\ ${ }^{2}$ School of Computer Science, University of Birmingham, United Kingdom
}

\begin{abstract}
We present a new Priestley-style topological duality for bounded N4-lattices, which are the algebraic counterpart of paraconsistent Nelson logic. Our duality differs from the existing one, due to Odintsov, in that we only rely on Esakia duality for Heyting algebras and not on the duality for De Morgan algebras of Cornish and Fowler. A major advantage of our approach is that for our topological structures we obtain a simple description, which can be easily extended to other algebras such as non-bounded N4-lattices and N4-lattices with modal operators.
\end{abstract}

Keywords: N4-lattices, paraconsistent Nelson logic, Priestley duality, twist-structures, Esakia duality

\section{Introduction}

N4-lattices are the algebraic semantics of paraconsistent Nelson logic, which was introduced in [1] as an inconsistency-tolerant counterpart of the betterknown logic of Nelson [2,3].

Paraconsistent Nelson logic combines interesting features of intuitionistic, classical and many-valued logics (e.g., Belnap-Dunn four-valued logic); recent work has shown that it can also be seen as one member of the wide family of substructural logics [4].

The work we present here is a contribution towards a better topological understanding of the algebraic counterpart of paraconsistent Nelson logic, namely a variety of involutive lattices called $\mathrm{N}_{4}$ lattices in [5]. A Priestley-style duality for these algebras was already introduced by Odintsov [6], the main difference between his approach and ours is that we only rely on Esakia duality for Heyting algebras [7], whereas [6] uses both Esakia duality and the duality for De Morgan algebras [8, 9]: as a consequence, the description of dual spaces that we obtain is, in our opinion, much simpler. Moreover, [6] only deals with N4-lattices whose lattice reduct is bounded, whereas we show how our treatment easily extends to the non-bounded case as well.

Outline of the paper. In the next section we introduce the abstract algebraic definition of N4-lattices and we state a fundamental result of Odintsov [10], namely that every N4-lattice can be represented through a concrete construction called twiststructure. We extend this to a categorial equivalence, which will allow us to work, for our duality, with a category of twist-structures instead of the category of N4-lattices as introduced in [6]. In Section 3 we recall the essentials of Esakia duality for Heyting algebras, on which we will build ours. Section 4 contains the main results. We show that our category of twist-structures (and, as a consequence, the category of N4-lattices) is dually equivalent to a category of Esakia spaces enriched with additional topological structure. Finally, in Section 5 we briefly discuss how to extend our duality to non-bounded N4-lattices and to N4-lattices expanded with modal operators.

\section{N4-lattices as twist-structures}

The abstract definition of N4-lattices [10, Definition $2.3]$ is rather obscure, but fortunately, as we will see, a more insightful description is available for these algebras thanks to the so-called twist-structure construction.

Definition 2.1. An N4-lattice is an algebra $\mathbf{B}=$ $\langle B, \wedge, \vee, \rightarrow, \sim\rangle$ such that:

(i) the reduct $\langle B, \wedge, \vee, \sim\rangle$ is a De Morgan lattice, i.e., a distributive lattice (with order $\leq$ ) equipped with a unary operation $\sim: B \rightarrow B$ (usually called negation) such that $\sim a=a$ and $\sim(a \vee b)=\sim a \wedge \sim b$,

(ii) the relation $\preceq$ defined as $a \preceq b$ iff $a \rightarrow b=$ $(a \rightarrow b) \rightarrow(a \rightarrow b)$, is a pre-ordering (i.e., reflexive and transitive),

(iii) the relation $\equiv$ defined as $a \equiv b$ iff $a \preceq b$ and $b \preceq a$ is a congruence w.r.t. $\wedge, \vee, \rightarrow$ and the quotient algebra $\mathbf{B}_{\bowtie}=\langle B, \wedge, \vee, \rightarrow\rangle / \equiv$ is a Brouwerian lattice ${ }^{1}$,

(iv) $\sim(a \rightarrow b) \equiv a \wedge \sim b$ for all $a, b \in B$,

(v) $a \leq b$ iff $a \preceq b$ and $\sim b \preceq \sim a$ for all $a, b \in B$.

$\mathbf{B}$ is said to be bounded if its lattice reduct is bounded (in which case we include the bounds as constants in the algebraic signature).

It is known that N4-lattices form a variety (hence, bounded N4-lattices are also a variety), which is

\footnotetext{
${ }^{1}$ A Brouwerian lattice is the 0 -free subreduct of a Heyting algebra. Brouwerian lattices are also known in the literature as generalized Heyting algebras [11], Brouwerian algebras [12], implicative lattices [5] and relatively pseudocomplemented lattices [3]. Some authors call "Brouwerian lattices" structures that are (lattice-theoretic) dual to ours.
} 
the equivalent algebraic semantics of paraconsistent Nelson logic (see [5]).

Condition (iii) of Definition 2.1 provides a canonical way of associating a Brouwerian lattice to a given N4-lattice. We are going to describe a method introduced in [10, Definition 2.1] to construct an N4lattice from a Brouwerian lattice, and we shall see that each N4-lattice is isomorphic to one obtained in this way.

Let $\mathbf{A}=\langle A, \wedge, \vee, \rightarrow, 1\rangle$ be a Brouwerian lattice. Consider the algebra $\mathbf{A}^{\bowtie}=\langle A \times A, \wedge, \vee, \rightarrow, \sim\rangle$ with operations defined as follows: for all $\langle a, b\rangle,\langle c, d\rangle \in$ $A \times A$,

$$
\begin{aligned}
\langle a, b\rangle \wedge\langle c, d\rangle & :=\langle a \wedge c, b \vee d\rangle \\
\langle a, b\rangle \vee\langle c, d\rangle & :=\langle a \vee c, b \wedge d\rangle \\
\langle a, b\rangle \rightarrow\langle c, d\rangle: & =\langle a \rightarrow c, a \wedge d\rangle \\
\sim\langle a, b\rangle & :=\langle b, a\rangle .
\end{aligned}
$$

It is easy to check that $\mathbf{A}^{\bowtie}$ is an N4-lattice. If $\mathbf{A}$ has a minimum element 0 , then by defining $\top:=\langle 1,0\rangle$ and $\perp:=\langle 0,1\rangle$ we obtain a bounded N4-lattice. Notice that the operations $\wedge, \vee, \rightarrow$ of $\mathbf{A}^{\bowtie}$ are defined component-wise just as in a direct product in the first component, while they are somehow "twisted" in the second one; this accounts for the name twist-structure over $\mathbf{A}$ for the algebra $\mathbf{A}^{\bowtie}$ used, e.g., in [10].

Although the above construction produces an N4lattice, not all N4-lattices are isomorphic to one constructed in this way. To achieve this, we need the following refinement.

Given a Brouwerian lattice $\mathbf{A}$, let $D(\mathbf{A}):=\{a \vee$ $(a \rightarrow b): a, b \in A\}$ be the set of its dense elements ${ }^{2}$. If $\mathbf{A}$ has a minimum 0 (i.e., if $\mathbf{A}$ "is" in fact a Heyting algebra), then the dense elements can also be obtained as follows: $D(\mathbf{A})=\{a \vee \neg a: a \in A\}=$ $\{a \in A: \neg \neg a=1\}$ where $\neg$ is the Heyting negation of $\mathbf{A}$, i.e., $\neg a:=a \rightarrow 0$. Now consider a lattice filter $\nabla \subseteq A$ such that $D(\mathbf{A}) \subseteq \nabla$ and an arbitrary (non-empty) lattice ideal $\Delta \subseteq A$. Then the set

$$
B:=\{\langle a, b\rangle \in A \times A: a \vee b \in \nabla, a \wedge b \in \Delta\}
$$

is closed under the operations $\wedge, \vee, \rightarrow, \sim$ of $\mathbf{A}^{\bowtie}$ and thus $\langle B, \wedge, \vee, \rightarrow, \sim\rangle$ is an N4-lattice. Following [10], we denote this algebra by $T w(\mathbf{A}, \nabla, \Delta)$.

The twist-structure construction described above is very similar to the one used in [13] to represent Brouwerian bilattices, the main differences being the following:

- The algebraic signature of Brouwerian bilattices includes the above-defined operations together with two additional ones, $\otimes$ and $\oplus$, defined by $\langle a, b\rangle \otimes\langle c, d\rangle:=\langle a \wedge c, b \wedge d\rangle$ and $\langle a, b\rangle \oplus\langle c, d\rangle:=\langle a \vee c, b \vee d\rangle$.

- As a consequence, every Brouwerian bilattice $\mathbf{B}$ corresponds to a twist-structure $\operatorname{Tw}(\mathbf{A}, \nabla, \Delta)$

\footnotetext{
${ }^{2}$ It is easy to check that $D(\mathbf{A})$ is a lattice filter, which we may call the filter of dense elements of $\mathbf{A}$.
}

whose carrier set is the whole direct product $A \times A$, which means that $\nabla=\Delta=A$.

Brouwerian bilattices can thus be viewed as special N4-lattices (and, conversely, N4-lattices correspond to subreducts of Brouwerian bilattices: see [13, p. 130]). These considerations imply that the topological duality we develop in the next sections also applies to Brouwerian bilattices. Moreover, since in this particular case $\nabla$ and $\Delta$ do not play any role, a duality can be obtained as a straightforward application of Esakia duality. Indeed, a duality for Brouwerian bilattices has already been introduced in [14, Section 3.4], drawing on Odintsov's duality for N4-lattices.

In order to show that any N4-lattice can be obtained as $T w(\mathbf{A}, \nabla, \Delta)$ for a suitable choice of $(\mathbf{A}, \nabla, \Delta)$, we define, for an arbitrary N4-lattice $\mathbf{B}$,

$$
\nabla(\mathbf{B}):=\{[a \vee \sim a]: a \in B\}
$$

where $[b]$ denotes the equivalence class of $b \in B$ modulo the relation $\equiv$ introduced in Definition 2.1. Similarly, we let

$$
\Delta(\mathbf{B}):=\{[a \wedge \sim a]: a \in B\} .
$$

It is not difficult to check that $\nabla(\mathbf{B})$ is a lattice filter of the Brouwerian lattice $\mathbf{B}_{\bowtie}=\langle B, \wedge, \vee, \rightarrow\rangle / \equiv$ which contains the dense elements of $\mathbf{B}_{\bowtie}$, and that $\Delta(\mathbf{B})$ is an ideal of $\mathbf{B}_{\bowtie \text {. Thus, we can construct }}$ the N4-lattice $T w\left(\mathbf{B}_{\bowtie}, \nabla(\mathbf{B}), \Delta(\mathbf{B})\right)$, which is indeed isomorphic to our original $\mathbf{B}[10$, Corollary $3.2]$.

Proposition 2.2. Every N4-lattice (bounded N4-lattice) $\mathbf{B}$ is isomorphic to the algebra $T w\left(\mathbf{B}_{\bowtie}, \nabla(\mathbf{B}), \Delta(\mathbf{B})\right) \quad$ with $\quad \mathbf{B}_{\bowtie} \quad$ a Brouwerian lattice (Heyting algebra), through the map $j_{\mathbf{B}}: B \rightarrow B / \equiv \times B / \equiv$ defined, for all $a \in B$, as $j_{\mathbf{B}}(a):=\langle[a],[\sim a]\rangle$.

Thus, any (bounded) N4-lattice can be associated to a triple of the form $(\mathbf{A}, \nabla, \Delta)$ with $\mathbf{A}$ a (bounded) Brouwerian lattice and $\nabla, \Delta$, respectively, a filter and an ideal of $\mathbf{A}$. We are going to see that $j_{\mathbf{B}}$ is in fact the unit of a categorical equivalence between two naturally associated categories.

The category N4 has as objects N4-lattices and as morphisms algebraic N4-lattice homomorphisms. On the other side of our equivalence, the category Twist has as objects triples $\mathcal{A}=(\mathbf{A}, \nabla, \Delta)$ with $\mathbf{A}$ a Brouwerian lattice, $\nabla \subseteq A$ a lattice filter containing the dense elements $D(\overline{\mathbf{A}})$, and $\Delta \subseteq A$ an ideal. We call objects in this category twist-structures, but notice that we view them just as triples $(\mathbf{A}, \nabla, \Delta)$, not as the product algebra $T w(\mathbf{A}, \nabla, \Delta)$ defined above. A morphism between twist-structures $\mathcal{A}_{1}, \mathcal{A}_{2}$ is a Brouwerian lattice homomorphism $h: \mathbf{A}_{1} \rightarrow \mathbf{A}_{2}$ such that $h\left[\nabla_{1}\right] \subseteq \nabla_{2}$ and $h\left[\Delta_{1}\right] \subseteq \Delta_{2}$. Twiststructures with twist-structure morphisms form a category, where the composition of morphisms is set-theoretical. 
We proceed to define functors $T: \mathrm{N} 4 \rightarrow$ Twist and $N$ : Twist $\rightarrow \mathrm{N} 4$ that will allow us to prove the equivalence between the two categories.

Given an N4-lattice $\mathbf{B}$, we let $T(\mathbf{B})$ := $\left(\mathbf{B}_{\bowtie}, \nabla(\mathbf{B}), \Delta(\mathbf{B})\right)$. If $f: \mathbf{B}_{\mathbf{1}} \rightarrow \mathbf{B}_{\mathbf{2}}$ is an N4morphism, we define $T(f):\left(\mathbf{B}_{\mathbf{1}}\right)_{\bowtie} \rightarrow\left(\mathbf{B}_{\mathbf{2}}\right)_{\bowtie}$ as $T(f)\left([a]_{\equiv_{1}}\right):=[f(a)]_{\equiv_{2}}$, where $[a]_{\equiv_{1}}$ is the equivalence class of $a \in B_{1}$ modulo the relation introduced in Definition 2.1 and likewise $[b]_{\equiv_{2}} \in B_{2} / \equiv_{2}$ for all $b \in B_{2}$. Then, $T(f)$ is a Twist-morphism from $\left(\mathbf{B}_{\mathbf{1}}\right)_{\bowtie}$ to $\left(\mathbf{B}_{\mathbf{2}}\right)_{\bowtie}$ satisfying $T(f) \circ \alpha_{1}=\alpha_{2} \circ f$, where $\alpha_{i}: \mathbf{B}_{i} \rightarrow \mathbf{B}_{i} / \equiv$ is defined by $\alpha_{i}(a):=[a]_{\equiv_{i}}$ for all $a \in B_{i}$. It is straightforward to check that $T$ is indeed a functor.

Conversely, for $\mathcal{A}=(\mathbf{A}, \nabla, \Delta) \in$ Twist, we let $N(\mathcal{A}):=T w(\mathbf{A}, \nabla, \Delta)$. We know by Proposition 2.2 that $T w(\mathbf{A}, \nabla, \Delta)$ is an N4-lattice. For a morphism $h: \mathcal{A}_{1} \rightarrow \mathcal{A}_{2}$ between twist-structures $\mathcal{A}_{1}, \mathcal{A}_{2}$, we define the map $N(h): N\left(\mathcal{A}_{1}\right) \rightarrow N\left(\mathcal{A}_{2}\right)$, for all $a, b \in A_{1}$, as $N(h)\langle a, b\rangle:=\langle h(a), h(b)\rangle$. It is easy to see that this map is well defined, that is, if $\langle a, b\rangle \in N\left(\mathcal{A}_{1}\right)$, then $\langle h(a), h(b)\rangle \in N\left(\mathcal{A}_{2}\right)$, and that it is an N4-lattice homomorphism. As before, it is easy to see that we have a functor $N$ : Twist $\rightarrow$ N4.

For any N4-lattice $\mathbf{B}$, by Proposition 2.2 we have an algebraic isomorphism $j_{\mathbf{B}}: \mathbf{B} \cong N(T(\mathbf{B}))$. This implies that $j_{\mathbf{B}}$ is an isomorphism in the category N4.

Observe, that for each twist-structure $\mathcal{A}$, we have $\langle a, a \rightarrow b\rangle \in N(\mathcal{A})$ for any $a \in A$ and any $b \in \Delta$. That is, $\pi_{1}[N(\mathcal{A})]=A$. Denoting, for all $a, b, a^{\prime}, b^{\prime} \in A$, by $\left[\left\langle a, a^{\prime}\right\rangle\right],\left[\left\langle b, b^{\prime}\right\rangle\right]$ the equivalence classes of $\left\langle a, a^{\prime}\right\rangle,\left\langle b, b^{\prime}\right\rangle$ modulo the equivalence relation of Definition 2.1, we have $\left[\left\langle a, a^{\prime}\right\rangle\right]=\left[\left\langle b, b^{\prime}\right\rangle\right]$ if and only if $a=b[10$, Proposition 2.1]. Thus, the map $\eta_{\mathcal{A}}: \mathcal{A} \rightarrow T(N(\mathcal{A}))$ given by $\eta_{\mathcal{A}}(a):=$ $\left\{\left\langle a, a^{\prime}\right\rangle \in N(\mathcal{A})\right\}=\left[\left\langle a, a^{\prime}\right\rangle\right]$ for all $a \in A$ is welldefined.

In this way we obtain the following.

Proposition 2.3. For any twist-structure $\mathcal{A}$, the map $\eta_{\mathcal{A}}: \mathcal{A} \rightarrow T(N(\mathcal{A}))$ is an isomorphism in the category Twist.

Proof. It follows from the above considerations that $\eta_{\mathcal{A}}$ is a bijection whose inverse is precisely $\pi_{1}$. Let us check that $\eta_{\mathcal{A}}$ is a Brouwerian lattice homomorphism. Let $a, b \in A$. Then $\eta_{\mathcal{A}}(a) \wedge \eta_{\mathcal{A}}(b)=$ $\left[\left\langle a, a^{\prime}\right\rangle\right] \wedge\left[\left\langle b, b^{\prime}\right\rangle\right]=\left[\left\langle a, a^{\prime}\right\rangle \wedge\left\langle b, b^{\prime}\right\rangle\right]=\left[\left\langle a \wedge b, a^{\prime} \vee b^{\prime}\right\rangle\right]$. But $\left[\left\langle a \wedge b, a^{\prime} \vee b^{\prime}\right\rangle\right]=[\langle a \wedge b, c\rangle]$ for any $c \in A$ such that $\langle a \wedge b, c\rangle \in N(\mathcal{A})$, so in particular we have $\left[\left\langle a \wedge b, a^{\prime} \vee b^{\prime}\right\rangle\right]=\eta_{\mathcal{A}}(a \wedge b)$. A similar reasoning establishes the cases of the other connectives. To prove that $\eta_{\mathcal{A}}[\nabla]=\nabla(N(\mathcal{A}))$, it is sufficient to observe that

$$
\begin{aligned}
\nabla(N(\mathcal{A})) & =\{[\langle a, b\rangle \vee \sim\langle a, b\rangle]:\langle a, b\rangle \in N(\mathcal{A})\} \\
& =\{[\langle a \vee b, a \wedge b\rangle]: a \vee b \in \nabla, a \wedge b \in \Delta\} \\
& =\{[\langle c, d\rangle]: c \in \nabla, d \in \Delta, d \leq c\} \\
& =\{[\langle c, d\rangle]: c \in \nabla\} \\
& =\eta_{\mathcal{A}}[\nabla] .
\end{aligned}
$$

The proof that $\eta_{\mathcal{A}}[\Delta]=\Delta(N(\mathcal{A}))$ is analogous.

Checking that the required diagrams commute is at this point just routine.

Proposition 2.4. Let $f: \mathbf{B}_{\mathbf{1}} \rightarrow \mathbf{B}_{\mathbf{2}}$ be an N4morphism. Then $N(T(f)) \circ j_{\mathbf{B}_{1}}=j_{\mathbf{B}_{2}} \circ f$.

Proof. For any $a \in B_{1}$,

$$
\begin{aligned}
N(T(f)) \circ j_{\mathbf{B}_{1}}(a) & =N(T(f))\left(\left\langle[a]_{\equiv_{1}},[\sim a]_{\equiv_{1}}\right\rangle\right) \\
& =\left\langle T(f)\left([a]_{\equiv_{1}}\right), T(f)\left([\sim a]_{\equiv_{1}}\right)\right\rangle \\
& =\left\langle[f(a)]_{\equiv_{2}},[f(\sim a)]_{\equiv_{2}}\right\rangle \\
& =\left\langle[f(a)]_{\equiv_{2}},[\sim f(a)]_{\equiv_{2}}\right\rangle \\
& =j_{\mathbf{B}_{2}} \circ f(a) .
\end{aligned}
$$

Proposition 2.5. Let $h: \mathcal{A}_{1} \rightarrow \mathcal{A}_{2}$ be a Twistmorphism. Then $T(N(h)) \circ \eta_{\mathcal{A}_{1}}=\eta_{\mathcal{A}_{2}} \circ h$.

Proof. For any $a \in A_{1}$,

$$
\begin{aligned}
T(N(h)) \circ \eta_{\mathcal{A}_{1}}(a) & =T(N(h))\left(\left[\left\langle a, a^{\prime}\right\rangle\right]_{\equiv_{1}}\right) \\
& =\left[N(h)\left(\left\langle a, a^{\prime}\right\rangle\right)\right]_{\equiv_{2}} \\
& =\left[\left\langle h(a), h\left(a^{\prime}\right)\right\rangle\right]_{\equiv_{2}} \\
& =\left[\left\langle h(a),(h(a))^{\prime}\right]_{\equiv_{2}}\right. \\
& =\eta_{\mathcal{A}_{2}} \circ h(a) .
\end{aligned}
$$

Recall that the equality $\left[\left\langle h(a), h\left(a^{\prime}\right)\right\rangle\right]_{\equiv_{2}}=$ $\left[\left\langle h(a),(h(a))^{\prime}\right]_{\equiv_{2}}\right.$ holds because $\left[\left\langle h(a), h\left(a^{\prime}\right)\right\rangle\right]_{\equiv_{2}}=$ $[\langle h(a), b\rangle]_{\equiv_{2}}$ for any $b \in A_{2}$ as long as $\langle h(a), b\rangle \in$ $N\left(\mathcal{A}_{2}\right)$.

The above propositions imply the announced equivalence result.

Theorem 2.6. Functors $T: \mathrm{N} 4 \rightarrow$ Twist and $N:$ Twist $\rightarrow \mathrm{N} 4$ establish a natural equivalence between the category N4 of N4-lattices and the category Twist of twist-structures over Brouwerian lattices.

It is straightforward to show that the above result restricts to an equivalence between the category $\mathrm{N}^{\perp}$ of bounded N4-lattices (with algebraic homomorphisms which preserve the bounds as morphisms) and the category Twist ${ }^{\perp}$ of twist-structures over bounded Brouwerian lattices (i.e., Heyting algebras), whose morphisms are defined as Twistmorphisms that also preserve the bounds. The picture is thus the following:

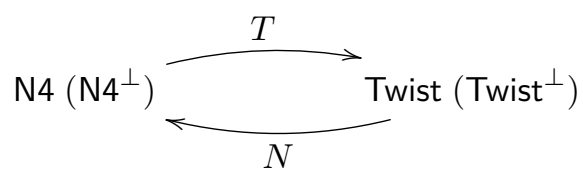

\section{Esakia duality}

We briefly recall the main definition and results of Esakia duality [7], on which we are going to build 
ours in the next section. We assume familiarity with Priestley duality for distributive lattices [15].

A Priestley space is a compact ordered space $\mathcal{X}=$ $\langle X, \tau, \leq\rangle$ such that, for every $x, y \in X$ with $x \not \leq y$, there is a clopen up-set $U$ such that $x \in U$ and $y \notin U$. A Priestley space is an Esakia space if, in addition, the down-set $\downarrow U$ of every clopen $U \subseteq X$ is clopen. The distributive lattice of clopen up-sets of an Esakia space forms a Heyting algebra when endowed with the following implication operation: for clopen up-sets $U, V \subseteq X$, we let $U \rightarrow V:=\{x \in$ $X: \uparrow x \cap U \subseteq V\}$. We denote this Heyting algebra by $A(\mathcal{X})$.

Conversely, to each Heyting algebra A corresponds an Esakia space $\left\langle X(\mathbf{A}), \tau_{\mathbf{A}}, \subseteq\right\rangle$ where $\langle X(\mathbf{A}), \subseteq\rangle$ is the poset of prime filters of $\mathbf{A}$ ordered by inclusion and $\tau_{\mathbf{A}}$ is the topology generated by the subbasis $\left\{\sigma_{\mathbf{A}}(a): a \in A\right\} \cup\left\{X(\mathbf{A})-\sigma_{\mathbf{A}}(a): a \in A\right\}$ where $\sigma_{\mathbf{A}}(a):=\{P \in X(\mathbf{A}): a \in P\}$.

Using the same definitions involved in Priestley duality, the maps $X($.$) and A($.$) can be extended to$ contravariant functors that establish a dual equivalence between (1) the category of Heyting algebras with algebraic homomorphisms as morphisms and (2) the category of Esakia spaces, whose morphisms are Esakia functions, defined as follows. An Esakia function is a map $f: X \rightarrow Y$ between Esakia spaces $\mathcal{X}, \mathcal{Y}$ which is continuous, order-preserving and such that $\uparrow_{Y} f(x) \subseteq f\left[\uparrow_{X} x\right]$ for every $x \in X$.

If $h: \mathbf{A}_{1} \rightarrow \mathbf{A}_{2}$ is a homomorphism of Heyting algebras, then the map $X(h): X\left(\mathbf{A}_{2}\right) \rightarrow X\left(\mathbf{A}_{1}\right)$ between the corresponding Esakia spaces defined by $X(h)(P)=h^{-1}[P]$ for every $P \in X\left(\mathbf{A}_{2}\right)$ is an Esakia function. Conversely, if $f: \mathcal{X} \rightarrow \mathcal{Y}$ is an Esakia function, the map $A(f): A(\mathcal{Y}) \rightarrow A(\mathcal{X})$ defined by $A(f)(U)=f^{-1}[U]$ for every clopen up-set $U \subseteq Y$ is a Heyting algebra homomorphism.

The natural transformations are given by the following families of morphisms. For a Heyting algebra $\mathbf{A}$, the map $\sigma_{\mathbf{A}}: \mathbf{A} \rightarrow A(X(\mathbf{A}))$ defined above is an isomorphism. For any Esakia space $\mathcal{X}$, the map $\epsilon_{X}: X \rightarrow X(A(\mathcal{X}))$ defined by $\epsilon_{X}(x)=\{U \in$ $A(\mathcal{X}): x \in U\}$ for every $x \in X$ is a homeomorphism and an order isomorphism.

\section{Duality for twist-structures}

We are now going to introduce a category of topological structures which we will prove to be equivalent to the category Twist $^{\perp}$ of twist-structures over Heyting algebras. At the end of the section we will sketch how to extend this duality to twist-structures over Brouwerian lattices.

The following property is going to be useful for the description of our topological structures.

Lemma 4.1. Let $P \subseteq A$ be a prime filter of a Brouwerian lattice (or Heyting algebra) $\mathbf{A}$ and $D(\mathbf{A})$ the filter of dense elements. Then $D(\mathbf{A}) \subseteq P$ if and only if $P$ is maximal in the poset $\langle X(\mathbf{A}), \subseteq\rangle$.
Proof. We prove that, if $P \subsetneq Q$ for some prime filter $Q$, then $Q=A$, so $Q$ is not prime. Assume that $P \subseteq Q$ and there is $a \in Q$ such that $a \notin P$. We claim that $b \in Q$ for an arbitrary element $b \in A$. By assumption we have $a \vee(a \rightarrow b) \in D(\mathbf{A}) \subseteq P$. Since $P$ is prime and $a \notin P$, we conclude that $a \rightarrow b \in$ $P \subseteq Q$. Now $a, a \rightarrow b \in Q$ imply that $a \wedge(a \rightarrow b)=$ $a \wedge b \in Q$. This means that $b \in Q$ as we claimed. Conversely, suppose that $P$ is a maximal element of the poset of prime filters of $\mathbf{A}$. Let $a, b \in A$ and assume that $a \vee(a \rightarrow b) \notin P$. Consider the filter $F$ generated by $P \cup\{a\}$. Then $a \rightarrow b \notin F$. On the contrary there is $c \in P$ such that $c \wedge a \leq a \rightarrow b$. Then $c \leq a \rightarrow(a \rightarrow b)=a \rightarrow b$. It would thus follow that $a \rightarrow b \in P$, against our assumption. So there is a prime filter $Q$ such that $P \varsubsetneqq F \subseteq Q$ and $a \rightarrow b \notin Q$. Therefore $P$ is not maximal: a contradiction. Hence $D(\mathbf{A}) \subseteq P$.

From now on, unless otherwise specified, we consider only twist-structures over Heyting algebras. Let then $\mathcal{A}=(\mathbf{A}, \nabla, \Delta) \in$ Twist $^{\perp}$ and let $\left\langle X(\mathbf{A}), \tau_{\mathbf{A}}, \subseteq\right\rangle$ be the Esakia space of $\mathbf{A}$. We know from Priestley duality that there is a one-to-one correspondence between all lattice filters of $\mathbf{A}$ and the closed up-sets of $X(\mathbf{A})$, and similarly ideals of $\mathbf{A}$ correspond to open up-sets of $X(\mathbf{A})$. Then, using the isomorphism $\sigma_{\mathbf{A}}: \mathbf{A} \cong A(X(\mathbf{A}))$, the sets $\nabla, \Delta \subseteq A$ can be represented as follows. We define

$$
C_{\mathcal{A}}:=\bigcap_{a \in \nabla} \sigma_{\mathbf{A}}(a) \quad O_{\mathcal{A}}:=\bigcup_{a \in \Delta} \sigma_{\mathbf{A}}(a)
$$

which are, respectively, a closed up-set and an open up-set. It is also easy to check that

$$
\begin{aligned}
& C_{\mathcal{A}}=\{P \in X(\mathbf{A}): \nabla \subseteq P\} \\
& O_{\mathcal{A}}=\{P \in X(\mathbf{A}): P \cap \Delta \neq \emptyset\} .
\end{aligned}
$$

We can thus use whichever of the above definitions is more convenient. We notice that $C_{\mathcal{A}}$ is included in the set $\max (X(\mathbf{A}))$ of maximal elements of our Esakia space (which also implies, trivially, that $C_{\mathcal{A}}$ is an up-set). This follows from Lemma 4.1, because $P \in C_{\mathcal{A}}$ implies that $D(\mathbf{A}) \subseteq \nabla \subseteq P$. We use this insight to introduce formally the topological structures we will deal with.

Definition 4.2. An NE-space (for Nelson-Esakia) is a structure $\mathcal{X}=\langle X, \leq, \tau, C, O\rangle$ such that

(i) $\langle X, \leq, \tau\rangle$ is an Esakia space,

(ii) $C$ is a closed set such that $C \subseteq \max (X)$,

(iii) $O$ is an open up-set.

In order to view NE-spaces as a category, we adopt the following notion of morphism.

Definition 4.3. Let $\mathcal{X}_{1}=\left\langle X_{1}, \leq_{1}, \tau_{1}, C_{1}, O_{1}\right\rangle$ and $\mathcal{X}_{2}=\left\langle X_{2}, \leq_{2}, \tau_{2}, C_{2}, O_{2}\right\rangle$ be NE-spaces. An NEmorphism is a map $f: X_{1} \rightarrow X_{2}$ such that 
(i) $f$ is an Esakia function, i.e., $f$ is monotone, continuous and $\uparrow f(x) \subseteq f[\uparrow x]$ for every $x \in$ $X_{1}$,

(ii) $f\left[C_{1}\right] \subseteq C_{2}$,

(iii) $f^{-1}\left[O_{2}\right] \subseteq O_{1}$.

Given NE-spaces $\mathcal{X}_{1}, \mathcal{X}_{2}, \mathcal{X}_{3}$ and NE-morphisms $f: \mathcal{X}_{1} \rightarrow \mathcal{X}_{2}, g: \mathcal{X}_{2} \rightarrow \mathcal{X}_{3}$, it is easy to see that $g \circ f: \mathcal{X}_{1} \rightarrow \mathcal{X}_{3}$ is also a morphism. Moreover, the identity map on an NE-space is a morphism. Thus, we have a category NE-Sp of NE-spaces.

Definition 4.2 immediately implies that, for any twist-structure $\mathcal{A}=(\mathbf{A}, \nabla, \Delta), \quad X(\mathcal{A}):=$ $\left\langle X(\mathbf{A}), \tau_{\mathbf{A}}, \subseteq, C_{\mathcal{A}}, O_{\mathcal{A}}\right\rangle$ is an NE-space. Given a morphism of twist-structures $h: \mathcal{A}_{1} \rightarrow \mathcal{A}_{2}$, we define the map $X(h): X\left(\mathcal{A}_{2}\right) \rightarrow X\left(\mathcal{A}_{1}\right)$ as in Esakia duality, so $X(h)$ is obviously an Esakia function. Let us check that the other requirements of Definition 4.3 are also met.

Lemma 4.4. Let $h: \mathcal{A}_{1} \rightarrow \mathcal{A}_{2}$ be a morphism between twist-structures $\mathcal{A}_{1}=\left\langle\mathbf{A}_{1}, \nabla_{1}, \Delta_{1}\right\rangle$ and $\mathcal{A}_{2}=\left\langle\mathbf{A}_{2}, \nabla_{2}, \Delta_{2}\right\rangle$. Then $X(h): X\left(\mathcal{A}_{2}\right) \rightarrow X\left(\mathcal{A}_{1}\right)$ is an NE-morphism between the corresponding $N E$ spaces.

Proof. In order to see that $X(h)\left[C_{\mathcal{A}_{2}}\right] \subseteq C_{\mathcal{A}_{1}}$, assume $Q \in X(h)\left[C_{\mathcal{A}_{2}}\right]$, i.e., $Q \in h^{-1}\left[C_{\mathcal{A}_{2}}\right]$. This means that there is $Q^{\prime} \in X\left(\mathbf{A}_{2}\right)$ such that $\nabla_{2} \subseteq Q^{\prime}$ and $Q=h^{-1}\left[Q^{\prime}\right]$. Since $h$ is a morphism of twiststructures, we have $h\left[\nabla_{1}\right] \subseteq \nabla_{2}$. This implies that $\nabla_{1} \subseteq h^{-1}\left[\nabla_{2}\right] \subseteq h^{-1}\left[Q^{\prime}\right]=Q$. We conclude that $\nabla_{1} \subseteq Q$, which means that $Q \in C_{\mathcal{A}_{1}}$ as desired. Assume now that $P \in X(h)^{-1}\left[O_{\mathcal{A}_{1}}\right]$. This means that $X(h)(P)=h^{-1}[P] \in O_{\mathcal{A}_{1}}$. Then $h^{-1}[P] \cap \Delta_{1} \neq \emptyset$. Let $a \in A_{1}$ be such that $a \in h^{-1}[P] \cap \Delta_{1}$. We then have $h(a) \in P \cap h\left(\Delta_{1}\right)$. From the assumptions we have $P \cap h\left(\Delta_{1}\right) \subseteq P \cap \Delta_{2}$, so we obtain $h(a) \in P \cap \Delta_{2} \neq \emptyset$, which implies $P \in O_{\mathcal{A}_{2}}$ as required. Thus, $X(h)$ is indeed a morphism of NEspaces.

It follows from Esakia duality that the map $X$ preserves composition and identity maps. So we actually have a functor $X$ : Twist ${ }^{\perp} \rightarrow \mathrm{NE}-\mathrm{Sp}$. It remains to define a functor $A: \mathrm{NE}-\mathrm{Sp} \rightarrow$ Twist $^{\perp}$ in the opposite direction.

To each NE-space $\mathcal{X}=\langle X, \leq, \tau, C, O\rangle$ we associate a twist-structure in the following way. Recall that $A(\mathcal{X})$ is the Heyting algebra of clopen up-sets of $\mathcal{X}$. To the closed set $C$ we associate the following filter of $A(\mathcal{X}): \nabla_{C}:=\{U \in A(\mathcal{X}): C \subseteq U\}$. Similarly, to the open up-set $O$ we associate the following ideal of $A(\mathcal{X}): \Delta_{O}:=\{U \in A(\mathcal{X}): U \subseteq O\}$.

In order to ensure that $\nabla_{C}$ does indeed contain the dense elements of $A(\mathcal{X})$, it is enough to realize that condition (ii) of Definition 4.2 is equivalent to the following property: for every clopen up-set $U \in$ $A(\mathcal{X}), C \subseteq U \cup(\downarrow U)^{c}$. In fact, we have

$$
\max (X)=\bigcap\left\{U \cup(\downarrow U)^{c}: U \in A(\mathcal{X})\right\} .
$$

The above considerations imply that $\left\langle A(\mathcal{X}), \nabla_{C}, \Delta_{O}\right\rangle$ is a twist-structure. Thus, for every object $\mathcal{X} \in \mathrm{NE}-\mathrm{Sp}$, we have an object $A(\mathcal{X})=\left\langle A(\mathcal{X}), \nabla_{C}, \Delta_{O}\right\rangle \in$ Twist $^{\perp}$. Let us now look at morphisms.

Given NE-spaces $\mathcal{X}_{1}=\left\langle X_{1}, \leq_{1}, \tau_{1}, C_{1}, O_{1}\right\rangle$ and $\mathcal{X}_{2}=\left\langle X_{2}, \leq_{2}, \tau_{2}, C_{2}, O_{2}\right\rangle$, let $f: X_{1} \rightarrow X_{2}$ be an NE-morphism. We know from Esakia duality that the map $A(f): A\left(\mathcal{X}_{2}\right) \rightarrow A\left(\mathcal{X}_{1}\right)$ is a Heyting algebra homomorphism. Let us check that it is in fact a Twist $^{\perp}$-morphism (as defined in Section 2) from $\left\langle A\left(\mathcal{X}_{2}\right), \nabla_{C_{2}}, \Delta_{O_{2}}\right\rangle$ to $\left\langle A\left(\mathcal{X}_{1}\right), \nabla_{C_{1}}, \Delta_{O_{1}}\right\rangle$.

Lemma 4.5. Let $f: \mathcal{X}_{1} \rightarrow \mathcal{X}_{2}$ be an NE-morphism. Then $A(f): A\left(\mathcal{X}_{2}\right) \rightarrow A\left(\mathcal{X}_{1}\right)$ is a Twist $^{\perp}$-morphism.

Proof. We need to show that $A(f)\left[\nabla_{C_{2}}\right] \subseteq \nabla_{C_{1}}$ and $A(f)\left[\Delta_{O_{2}}\right] \subseteq \Delta_{O_{1}}$. Let $U \in A(f)\left[\nabla_{C_{2}}\right]$ and $V \in$ $\nabla_{C_{2}}$ be such that $U=A(f)(V)=f^{-1}[V]$. Since $V \in \nabla_{C_{2}}$ we have $C_{2} \subseteq V$. So $f^{-1}\left[C_{2}\right] \subseteq f^{-1}[V]$. Then, since $f\left[C_{1}\right] \subseteq C_{2}$, we have $C_{1} \subseteq f^{-1}\left[C_{2}\right]$. Therefore, $C_{1} \subseteq f^{-1}[V]$. Hence, $f^{-1}[V] \in \nabla_{C_{1}}$. Now let $U \in A(f)\left[\Delta_{O_{2}}\right]$ and assume that $V \in \Delta_{O_{2}}$ is such that $A(f)(V)=U$, so that $f^{-1}[V]=U$. Since $V \in \Delta_{O_{2}}$, we have $V \subseteq O_{2}$. Therefore, $U=$ $f^{-1}[V] \subseteq f^{-1}\left[O_{2}\right] \subseteq O_{1}$. Hence, $U \in \Delta_{O_{1}}$.

We thus have a functor $A: \mathrm{NE}-\mathrm{Sp} \rightarrow$ Twist $^{\perp}$ as required. We are now going to see that, for any twist-structure $\mathcal{A}$ and any NE-space $\mathcal{X}$, there are natural isomorphisms $\sigma_{\mathbf{A}}: \mathcal{A} \cong A(X(\mathcal{A}))$ and $\epsilon_{\mathcal{X}}: \mathcal{X} \cong X(A(\mathcal{X}))$.

Given a twist-structure $\mathcal{A}=(\mathbf{A}, \nabla, \Delta)$, consider the twist-structure associated with the dual space of $\mathcal{A}$, namely $\left\langle A(X(\mathcal{A})), \nabla_{C_{\mathcal{A}}}, \Delta_{O_{\mathcal{A}}}\right\rangle$. We know from Esakia duality that the map $\sigma_{\mathrm{A}}: A \rightarrow A(X(\mathcal{A}))$ is a Heyting algebra isomorphism. Thus, we only need to check that $\sigma_{\mathbf{A}}$ is indeed a Twist ${ }^{\perp}$-morphism. This follows from next lemma (we omit the subscript of $\sigma_{\mathbf{A}}$ when no ambiguity can arise).

Lemma 4.6. For any twist-structure $\mathcal{A}=$ $\langle\mathbf{A}, \nabla, \Delta\rangle$, the map $\sigma_{\mathbf{A}}: A \rightarrow A(X(\mathcal{A}))$ satisfies:

(i) $\sigma_{\mathbf{A}}[\nabla]=\nabla_{C_{\mathcal{A}}}$

(ii) $\sigma_{\mathbf{A}}[\Delta]=\Delta_{O_{\mathcal{A}}}$.

Proof. (i) Let $a \in \nabla$. Then $C_{\mathcal{A}} \subseteq \sigma(a)$, so $\sigma(a) \in$ $\nabla_{C_{\mathcal{A}}}$. Let now $\sigma(a) \in \nabla_{C_{\mathcal{A}}}$. Then $C_{\mathcal{A}} \subseteq \sigma(a)$. Suppose that $a \notin \nabla$. Let $P$ be a prime filter such that $\nabla \subseteq P$ and $a \notin P$. Then $P \in \bigcap_{b \in \nabla} \sigma(b)=C_{\mathcal{A}}$. So $P \in \sigma(a)$, a contradiction. Therefore $a \in \nabla$, hence $\sigma(a) \in \sigma[\nabla]$. (ii) Let $a \in \Delta$. Then $\sigma(a) \subseteq$ $O_{\mathcal{A}}$. Therefore, $\sigma(a) \in \Delta_{O_{\mathcal{A}}}$. Suppose now that $\sigma(a) \in \Delta_{O_{\mathcal{A}}}$. Then, $\sigma(a) \subseteq O_{\mathcal{A}}$. Suppose that $a \notin \Delta$. Let $P$ be a prime filter such that $a \in P$ and $P \cap \Delta=\emptyset$. Since $O_{\mathcal{A}}=\bigcup_{b \in \Delta} \sigma(b)$, we obtain that $P \notin O_{\mathcal{A}}$, a contradiction. We conclude that $a \in \Delta$ and $\sigma(a) \in \sigma[\Delta]$.

Conversely, given an NE-space $\mathcal{X}$, consider the NE-space corresponding to the twist-structure 
$A(\mathcal{X})$, i.e., $\left\langle X(A(\mathcal{X})), \subseteq, \tau_{\mathcal{A}}, C_{A(\mathcal{X})}, O_{A(\mathcal{X})}\right\rangle$. Recall that the map $\epsilon_{\mathcal{X}}: X \rightarrow X(A(\mathcal{X}))$ is an Esakia-homeomorphism between $\mathcal{X}=\langle X, \leq, \tau\rangle$ and $\left\langle X(A(\mathcal{X})), \subseteq, \tau_{A(\mathcal{X})}\right\rangle$. We check that $\epsilon_{\mathcal{X}}$ is an NEmorphism as well.

Lemma 4.7. Let $\mathcal{X}=\langle X, \leq, \tau, C, O\rangle$ be an $N E$ space. The map $\epsilon_{\mathcal{X}}: X \rightarrow X(\mathcal{A}(\mathcal{X}))$ satisfies:

(i) $\epsilon_{\mathcal{X}}[C]=C_{A(\mathcal{X})}$

(ii) $\epsilon_{\mathcal{X}}[O]=O_{A(\mathcal{X})}$.

Proof. (i) Let $x \in C$. We have to see that $\epsilon_{\mathcal{X}}(x) \in$ $\bigcap_{U \in \nabla_{C}} \sigma(U)$, i.e., that for every $U \in A(\mathcal{X})$ such that $C \subseteq U$, it holds that $U \in \epsilon_{\mathcal{X}}(x)$. Assume then $C \subseteq U \in A(\mathcal{X})$. Then $x \in U$, so $U \in \epsilon_{\mathcal{X}}(x)$. Conversely, assume $x \in U$ for every clopen up-set $U \supseteq C$. Since $C$ is a closed up-set, $C=\bigcap\{U \in A(\mathcal{X}): C \subseteq U\}$. Therefore, $x \in C$ and $\epsilon_{\mathcal{X}}(x) \in \epsilon_{\mathcal{X}}[C]$. (ii) For $x \in O$, we have to see that $\epsilon_{\mathcal{X}}(x) \in \bigcup_{U \in \Delta_{O}} \sigma(U)$, i.e., that $U \in \epsilon_{\mathcal{X}}(x)$ for some $U \in A(\mathcal{X})$ such that $U \subseteq O$. Suppose the contrary. Then, for every $U \in A(\mathcal{X})$ with $U \subseteq O$, it holds that $x \notin U$. It follows that $x \notin O$, a contradiction. Hence, $\epsilon_{\mathcal{X}}(x) \in \bigcup_{U \in \Delta_{O}} \sigma(U)$. Assume now $\epsilon_{\mathcal{X}}(x) \in \bigcup_{U \in \Delta_{O}} \sigma(U)$. Then there is a clopen up-set $U \subseteq O$ such that $x \in U$. Since $O$ is an open up-set, $O=\bigcup\{U \in A(\mathcal{X}): U \subseteq O\}$. Therefore, $x \in O$ and $\epsilon_{\mathcal{X}}(x) \in \epsilon_{\mathcal{X}}[O]$.

The fact that $\sigma_{\mathbf{A}}$ and $\epsilon_{\mathcal{X}}$ are natural follows immediately from Esakia duality. Thus, if $h: \mathcal{A}_{1} \rightarrow$ $\mathcal{A}_{2}$ is a morphism of twist-structures, then $\sigma_{\mathcal{A}_{2}} \circ h=$ $A(X(h)) \circ \sigma_{\mathcal{A}_{1}}$. Likewise, if $f: \mathcal{X}_{1} \rightarrow \mathcal{X}_{2}$ is a morphism of NE-spaces, then $\epsilon_{\mathcal{X}_{2}} \circ f=X(A(f)) \circ \epsilon_{\mathcal{X}_{1}}$. Joining these results, we obtain the announced dual equivalences.

Theorem 4.8. The functors $X:$ Twist $^{\perp} \rightarrow$ NE-Sp and $A: \mathrm{NE}-\mathrm{Sp} \rightarrow$ Twist $^{\perp}$ establish a dual equivalence between the category Twist $^{\perp}$ of twist-structures over Heyting algebras and the category NE-Sp of NE-spaces.

Corollary 4.9. The category $\mathrm{N}^{\perp}$ of bounded $\mathrm{N}_{4}$ lattices and the category NE-Sp of NE-spaces are dually equivalent via functors $X \circ T: \mathrm{N} 4^{\perp} \rightarrow \mathrm{NE}-\mathrm{Sp}$ and $N \circ A: \mathrm{NE}-\mathrm{Sp} \rightarrow \mathrm{N} 4^{\perp}$.

The figure below displays the equivalences established so far.

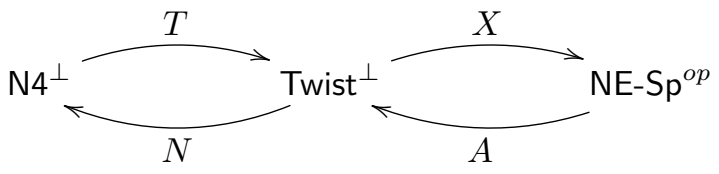

\section{Extending the duality}

As mentioned in the introduction, the duality presented above can be easily extended to twiststructures over Brouwerian lattices. All that is needed for this is to adapt Esakia duality to Brouwerian lattices. We sketch the main idea below.

Any Brouwerian lattice $\mathbf{A}=\langle A, \wedge, \vee, \rightarrow, 1\rangle$ can be extended to a Heyting algebra in the following way. Regardless of whether $\mathbf{A}$ has a minimum, we add a new one $0^{*}$, setting $0^{*} \leq a$ for all $a \in A \cup\left\{0^{*}\right\}$. This determines the behaviour of Heyting implication, because it must hold that $0^{*} \rightarrow a=1$ for all $a \in A \cup\left\{0^{*}\right\}$, and residuation implies that $a \rightarrow 0^{*}=\max \left\{b \in A \cup\left\{0^{*}\right\}: a \wedge b \leq 0^{*}\right\}$, which means that for $a \neq 0^{*}$ the only possible choice is $b=0^{*}$. Hence, we define

$$
a \rightarrow 0^{*}:= \begin{cases}0^{*} & \text { if } a \in A \\ 1 & \text { otherwise (i.e., if } \left.a=0^{*}\right) .\end{cases}
$$

In this way we obtain a Heyting algebra $\mathbf{A}^{*}$ with universe $A \cup\left\{0^{*}\right\}$, of which $\mathbf{A}$ is a $\{\wedge, \vee, \rightarrow, 1\}$ subalgebra. Notice that a map $h: \mathbf{A}_{1}^{*} \rightarrow \mathbf{A}_{2}^{*}$ is a Heyting algebra homomorphism if and only if the restriction $h \uparrow_{A_{1}}: \mathbf{A}_{1} \rightarrow \mathbf{A}_{2}$ is a Brouwerian lattice homomorphism. This implies that the category of Brouwerian lattices is equivalent to a full subcategory of the category of Heyting algebras.

If we now look at $X\left(\mathbf{A}^{*}\right)$, the Esakia space corresponding to $\mathbf{A}^{*}$, we may notice that $X\left(\mathbf{A}^{*}\right)$ has a greatest element, namely $A$, so that $A \in \sigma_{\mathbf{A}^{*}}(a)$ for every $a \in A$. The map $\sigma_{\mathbf{A} *}$ restricted to $A$ establishes an isomorphism between $\mathbf{A}$ and the algebra of non-empty clopen up-sets of $X\left(\mathbf{A}^{*}\right)$, which is a Brouwerian lattice. This makes it possible to recover our original $\mathbf{A}$ as the lattice of non-empty clopen up-sets of the Esakia dual of $\mathbf{A}^{*}$.

Thus, we can develop a duality between Brouwerian lattices and a category whose objects are Esakia spaces $\mathcal{X}=\langle X, \tau, \leq\rangle$ where the poset $\langle X, \leq\rangle$ has a maximum element $1_{X}$ and whose morphisms are Esakia functions $f: \mathcal{X} \rightarrow \mathcal{Y}$ such that $f\left(1_{X}\right)=1_{Y}$.

Once we have this, a duality for twist-structures $(\mathbf{A}, \nabla, \Delta)$ with $\mathbf{A}$ a Brouwerian lattice can be obtained by applying the same technique used in the previous sections to take care of the topological counterparts of $\nabla$ and $\Delta$. The dual category obtained in this way has as objects structures $\mathcal{X}=$ $\left\langle X, \leq, 1_{X}, \tau, C, O\right\rangle$ where $\left\langle X, \leq, 1_{X}, \tau\right\rangle$ is an Esakia space with maximum, $O$ is a non-empty open upset and $C$ is a non-empty closed up-set whose elements are maximal in $X-\left\{1_{X}\right\}$. The morphisms are simply NE-morphisms $f: X \rightarrow Y$ such that $f\left(1_{X}\right)=1_{Y}$.

We mention, as a topic for further investigation, another direction in which our duality might be extended. Recent works on modal expansions of Belnap-Dunn logic [16, 17, 18] consider N4-lattices expanded with modal operators. These enriched algebras are also representable as twist-structures $(\mathbf{A}, \nabla, \Delta)$, where $\mathbf{A}$ is a Brouwerian lattice (or a Heyting or a Boolean algebra) which is itself endowed with modal operators. A topological duality for these "modal twist-structures" (and, therefore, for N4-lattices with modal operators) might be de- 
veloped following the same ideas expounded in the previous sections, drawing on the existing duality theory for distributive lattices with modal operators (see, e.g., [19]). Besides its intrinsic interest, such an investigation is likely to shed further light on the semantics of modal expansions of BelnapDunn logic; it might, for instance, enable us to introduce a state-based semantics for the non-normal paraconsistent modal logic of [18].

\section{Acknowledgements}

We would like to thank Achim Jung for several discussions in which his topological expertise was very helpful. We are also indebted to an anonymous referee for pointing out a mistake in one proof, helping to simplyfy several others, and providing suggestions that helped to improve the text.

The research leading to these results has received funding from the People Programme (Marie Curie Actions) of the European Union's Seventh Framework Programme (FP7/2007-2013) under REA grant agreement PIEF-GA-2010-272737 and was also partially supported by the grants 2009SGR-1433 of the AGAUR of the Generalitat de Catalunya and MTM2011-25747 of the Spanish Ministerio de Ciencia e Innovación, which includes EU Feder funds.

\section{References}

[1] A. Almukdad and D. Nelson. Constructible falsity and inexact predicates. The Journal of Symbolic Logic, 49(1):231-233, 1984.

[2] D. Nelson. Constructible falsity. The Journal of Symbolic Logic, 14:16-26, 1949.

[3] H. Rasiowa. An algebraic approach to nonclassical logics, volume 78 of Studies in Logic and the Foundations of Mathematics. NorthHolland, Amsterdam, 1974.

[4] M. Spinks and R. Veroff. Paraconsistent constructive logic with strong negation is a contraction-free relevant logic. In preparation.

[5] S. P. Odintsov. Algebraic semantics for paraconsistent Nelson's logic. Journal of Logic and Computation, 13(4):453-468, 2003.

[6] S. P. Odintsov. Priestley duality for paraconsistent nelson's logic. Studia Logica, 96(1):65-93, 2010.

[7] L. Esakia. Topological Kripke models. Soviet Math. Dokl., 15:147-151, 1974.

[8] W. H. Cornish and P. R. Fowler. Coproducts of De Morgan algebras. Bull. Austral. Math. Soc., 16(1):1-13, 1977.

[9] W. H. Cornish and P. R. Fowler. Coproducts of Kleene algebras. J. Austral. Math. Soc. Ser. A, 27(2):209-220, 1979.

[10] S. P. Odintsov. On the representation of N4lattices. Studia Logica, 76(3):385-405, 2004.
[11] R. Cignoli and A. Torrens. Free Algebras in Varieties of Glivenko MTL-algebras Satisfying the Equation $2\left(x^{2}\right)=(2 x)^{2}$. Studia Logica, 83(1-3):157-181, 2006.

[12] B. A. Davey. Dualities for equational classes of Brouwerian algebras and Heyting algebras. Transactions of the Americal Mathematical Society, 221(1):119-146, 1976.

[13] F. Bou, R. Jansana, and U. Rivieccio. Varieties of interlaced bilattices. Algebra Universalis, 66(1):115-141, 2011.

[14] A. Jung and U. Rivieccio. Priestley duality for bilattices. Studia Logica, 100(1-2):223-252, 2012.

[15] B. A. Davey and H. A. Priestley. Introduction to lattices and order. Cambridge University Press, New York, second edition, 2002.

[16] S. P. Odintsov and H. Wansing. Modal logics with Belnapian truth values. Journal of $A p$ plied Non-Classical Logics, 20:279-301, 2010.

[17] S. P. Odintsov and E. I. Latkin. BK-lattices. Algebraic semantics for Belnapian modal logics. Studia Logica, 100(1-2):319-338, 2012.

[18] U. Rivieccio. Paraconsistent modal logics. Electronic Notes in Theoretical Computer Science, 278:173-186, 2011.

[19] R. Goldblatt. Varieties of Complex Algebras. Annals of Pure and Applied Logic, 44(3):173242, 1989. 